\title{
Understanding elementary mathematics teacher education through the characteristics and activities of preservice teachers, elementary mathematics specialists, and mathematics teacher educators
}

\author{
Gwendolyn M. Lloyd ${ }^{1}$
}

Published online: 8 March 2018

(C) Springer Science+Business Media B.V., part of Springer Nature 2018

Researchers who study elementary mathematics teacher education focus on participants' experiences in a multitude of educational contexts, apply different conceptual and theoretical perspectives and empirical methods, and investigate unique aspects of teacher development related to mathematics teaching and learning. This variety is reflected in the four articles of this issue of the Journal of Mathematics Teacher Education. These articles include reports about preservice teachers [PSTs] participating in problem-solving activities in an elementary mathematics methods course, certified teachers enrolled in two elementary mathematics specialist [EMS] programs, and mathematics teacher educators [MTEs] teaching content courses. In their studies, researchers drew upon frameworks and perspectives on mathematical understanding and problem-solving strategies; content knowledge, beliefs, attitudes, and instructional practices; effective professional development; and teaching as design. They used varied data collection tools and analytic methods to investigate PSTs' strategies in solving an intentionally misleading problem (S. Boote and D. Boote); the impact of EMS programs on teachers' knowledge, beliefs, attitudes, and instructional practices (Swars et al.; Kutaka et al.); and MTEs' perspectives on content course designs ( $\mathrm{Li}$ and Superfine). These researchers offer the field new insights into the participants and activities of elementary mathematics teacher education.

How PSTs engage in the kinds of mathematical activities that they will one day facilitate with children is a worthwhile consideration for research. In "ABC Problem in Elementary Mathematics Education: Arithmetic Before Comprehension," Stacy Boote and David Boote describe a study of elementary PSTs' solutions to an intentionally misleading mathematics problem (the Log Problem). This non-routine problem invites the solver to use surface features - the immediately accessible numbers in the problem statementrather than subtle but meaningful details from the problem context. Reliance on the

Gwendolyn M. Lloyd

1loyd@psu.edu

1 The Pennsylvania State University, University Park, PA, USA 
numbers from the text of an intentionally misleading problem will result in an incorrect solution. Drawing on a theory of mathematical understanding that contrasts direct translation and problem model strategies, the researchers aimed to identify patterns in 121 PSTs' use of models to solve the intentionally misleading problem. PSTs' problemsolving journals, showing their work on the Log Problem, were analyzed quantitatively and qualitatively. Boote and Boote found that most PSTs' solutions involved a direct translation strategy. Correct solutions were much more likely to involve multiple diagrams and equations; however, multiple models did not guarantee a correct response, as many PSTs modeled the problem using direct translation. When PSTs made corrections to initial strategies, they tended to be assisted by visual feedback from diagrams. These interesting results have implications for mathematics teacher educators who have opportunities to engage PSTs in problem-solving activities. Bringing attention to problem comprehension and modeling in teacher education courses has potential to challenge PSTs' long held beliefs and habits.

Two teams of researchers report about investigations into teacher development in EMS programs. In the USA, where elementary teachers are prepared as generalists and typically have limited mathematics backgrounds, the role of EMS is filled by teachers who provide expertise in mathematics teaching and learning to a school, district, or larger professional community. An important question is how to prepare teachers for this fairly new role of EMS. Susan Swars, Stephanie Smith, Marvin Smith, Jody Carothers, and Kayla Myers report about the impact of a K-5 Mathematics Endorsement Program on teachers' knowledge, beliefs, and practices. Similarly, Traci Kutaka et al. describe the impact of a K-3 EMS program on teachers' knowledge, attitudes, and beliefs (teacher variables that are considered to be closely related to classroom practices). Both of these studies contribute to an emerging body of research about EMS programs and the development of EMSs.

In "The Preparation Experiences of Elementary Mathematics Specialists: Examining Influences on Beliefs, Content Knowledge, and Teaching Practices," Swars et al. write about a mixed methods study of the development of 32 elementary teachers, each with at least one year of classroom experience, enrolled in a two-semester graduate level EMS program. The program includes four intentionally designed mathematics courses with integrated pedagogy, one mathematics education practicum course, and additional graduate coursework. The researchers aimed to identify changes in teachers' mathematical beliefs, specialized content knowledge, and classroom teaching practices. Data were collected via belief surveys, a knowledge assessment, and teaching observations at multiple points in the teachers' enrollment in the program. Interviews were also conducted with six teachers. Through their analysis, the researchers detected shifts in teachers' pedagogical beliefs (mainly during the first half of the program) and teaching efficacy beliefs (mainly during the second half of the program). It seems possible, as Swars and her colleagues suggest, that teachers needed an extended time to develop confidence in their ability to influence student learning. An interesting relationship was noted between strong teaching efficacy and cognitively-oriented pedagogical beliefs. In addition, the researchers found that teachers' specialized content knowledge and mathematics teaching practices improved over the course of the EMS program. Based on analysis of teaching observations, they noted key areas for future growth in classroom practices (e.g., opportunities for children to make conjectures), valuable information for this program and other professional development efforts. The authors suggest the need for future studies of how EMS programs can offer long-lasting opportunities for teachers to change classroom practices.

The development of EMSs is also the focus of Kutaka et al.'s article, "Examining Change in K-3 Teachers' Mathematical Knowledge, Attitudes, and Beliefs: The Case of 
Primarily Math." These researchers sought to examine changes in teachers' mathematical knowledge for teaching, attitudes toward mathematics learning, and beliefs about teaching and learning through participation in an EMT program, Primarily Math. This program includes six graduate level courses over a 13-month period. Three courses focus on mathematical knowledge for teaching, and three focus on pedagogy and child development. Kutaka and her colleagues provide a detailed description of the views and principles that guided the design of the course activities in the program. The study's research design included comparisons of 126 teachers' scores on knowledge, attitudes, and beliefs instruments at the beginning and end of the program as well as comparisons to a matched group of 92 non-Primarily Math teachers. The first cohort of teachers showed improvements in their mathematical knowledge for teaching in two areas, with continued growth after coursework completion. Similar patterns of change emerged for the next two cohorts, and overall the Primarily Math teachers showed more growth than comparison group teachers. The researchers reported positive results with respect to teachers' attitudes toward learning mathematics (confidence, motivation, and anxiety) and teachers' beliefs. Lower levels of teacher-centered beliefs and higher levels of student-centered beliefs were reported after completing the program, and these changes were also evident in comparison with non-EMS teachers. The findings of this study, namely the statistically significant changes in Primarily Math teachers' knowledge, attitudes, and beliefs, offer noteworthy advancements in the field's understanding of the preparation of teachers as EMSs.

Just as understanding the development of teachers is important, so too is investigating the work of the MTEs who create and facilitate learning opportunities for teachers. Mathematics content courses for PSTs, a component of elementary mathematics teacher education with a limited research base, provide the context for Wenjuan Li and Alison Castro Superfine's study of MTEs' design goals. In "Mathematics Teacher Educators' Perspectives on Their Design of Content Courses for Elementary Preservice Teachers," these authors take a teaching as design perspective to investigate the instructional goals of MTEs, how MTEs' meet their goals, and the challenges they face in doing so. Six MTEs, with varying years of experience teaching content courses for PSTs and teaching in K-12 classrooms, participated in semi-structured interviews that were analyzed individually and across cases. The researchers found that all six MTEs had design goals for their content courses that reflected a learner-centered approach focused on the development of conceptual understanding. They viewed their courses as characterized by activities with worthwhile mathematical tasks, connections to teaching practice, and collaborative work. Several challenges were shared by MTEs, including managing PSTs' diverse mathematical backgrounds. An interesting contribution of the study is the recognition that MTEs' work, while sharing some qualities, is importantly different from the work of K-12 teachers. In addition to suggesting fruitful areas of professional development for MTEs, the researchers speculate that with improved understandings of MTEs' design of content courses, we may also gain new insights into preservice teachers' learning experiences in teacher preparation.

The reports in this issue of JMTE illustrate the value of ongoing research in elementary mathematics teacher education. By investigating characteristics and activities of PSTs, EMSs, and MTEs, these researchers make important contributions to our understandings, but also raise questions and expose areas in need of further exploration. Promising areas for future study include relationships between PSTs' problem-solving habits and beliefs about student learning, long-term impacts of particular EMS program characteristics on teachers' growth, and professional development activities that support MTEs in providing effective instruction in mathematics content courses. Research in these areas has potential to extend 
the results of the studies reported in this issue and further enhance the field's knowledge about the development of teachers and teacher educators. 